\title{
Photo-induced separation of chiral isomers in a classical buffer gas
}

\author{
B. Spivak and A. V. Andreev \\ Physics Department of the University of Washington, Seattle WA 98195
}

(Dated: August 22, 2008)

\begin{abstract}
We develop a theory of photo-induced drift of chiral molecules or small particles in classical buffer gases. In the absence of a magnetic field there exists a flux of chiral molecules, provided the electromagnetic field is circularly polarized. It has opposite signs for different chiral isomers. In the presence of a magnetic field the flux can be also induced by a linearly polarized (or unpolarized) electromagnetic field. The magnitude of the flux is not proportional to either linear or orbital momentum of the electromagnetic field.
\end{abstract}

PACS numbers: 37.10.Vz, 42.62.Be, 47.45.Ab

In this article we develop a theory of photo-induced transport of chiral molecules (or small chiral particles) diluted in a classical buffer gas. This effect can be important for separation of different isomers of chiral molecules and for the theory of optically actuated molecular motors (see for example [1, 2, 3, 4, 5]).

Consider a solution of molecules of definite chirality in a buffer gas in the presence of an electromagnetic radiation with frequency $\omega$ and polarization tensor $P_{i j}=\left\langle E_{i} E_{j}^{*}\right\rangle$, where $\mathbf{E}$ is electric field and $\langle\ldots\rangle$ denotes time averaging. We define the chiral current $\mathbf{j}_{c}$ as the part of molecular current that changes sign when the molecules are replaced by stereo-isomers of opposite chirality. We study effects whose magnitudes are not proportional to either linear or angular momentum of light, i.e. we work in the approximation where no force or torque is exerted by the electromagnetic radiation on the medium. This approximation is valid as long as the thermal angular momentum of the molecules is larger than $\hbar$. In this case in the absence of a magnetic field, by symmetry, the chiral flux density is nonzero only if the electromagnetic field is circularly polarized,

$$
j_{c, k}=\alpha_{c} \epsilon_{i j k} P_{i j} .
$$

Here $\epsilon_{i j k}$ is the antisymmetric tensor, and the coefficient $\alpha_{c}$ has opposite signs for molecules of different chirality. It is clear from Eq. (1) that when the direction of circular polarization is reversed the chiral current changes sign.

Although the photo-induced flux of chiral molecules exists both in gases and liquids the microscopic mechanisms of the effect in these two cases are different. In this article we focus on the case of gases, where all relevant mean free paths (for both buffer gas and chiral molecules) are bigger than sizes of the molecules. In this case the effect can be analyzed using the Boltzmann kinetic equation.

We assume that optical transitions take place between the ground state and an exited state of the chiral molecules denoted by indices $g$ and $e$ respectively, the energy difference between them being $\hbar \omega_{0}$.

Let us first illustrate the origin of the effect by considering a toy model in which the chiral molecules are constrained to rotate about a single axis which coincides

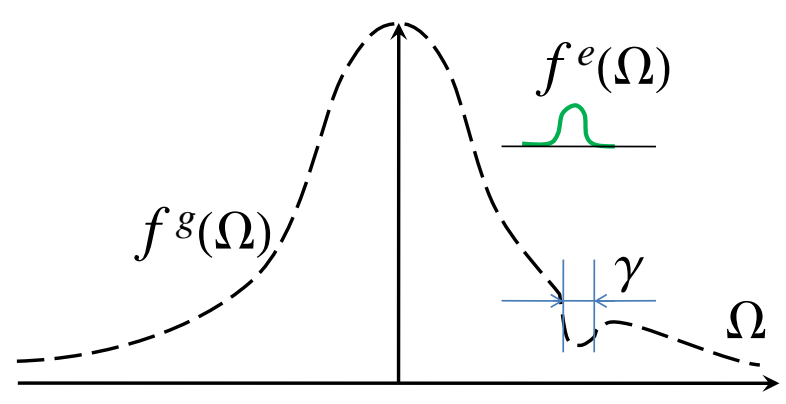

FIG. 1: (a) Schematic drawing of the dependence of the distribution function of chiral molecules on their angular velocity along the wavevector of light.

with the direction of propagation of light. The frequency of the circularly polarized radiation in the reference frame co-rotating with the molecule is shifted by the rotation frequency of the molecule, $\Omega$. As a result the probabilities of light absorption by molecules rotating clockwise and counterclockwise are different. Consequently the distribution function of the molecules becomes asymmetric with respect to the angular velocity: a hole will occur in the distribution function of the ground state molecules $f^{g}(\Omega)$ at certain values of the angular velocity, while a peak will occur in the exited state distribution $f^{e}(\Omega)$ (see Fig. 1). Thus the populations of molecules in the excited and ground states acquire opposite angular momenta. A fundamental property of collisions of the chiral molecules with the buffer gas is that they transfer rotation of chiral molecules into translation. Since the scattering cross-sections for the excited and ground states are generally different this will result in the net flux of the chiral molecules. Momentum conservation during collisions implies that a net momentum in the direction opposite to the flux is imparted to the buffer gas. In some aspects this effect is similar to the light-induced drift of atoms considered in Refs. 6, 7, 8, 9,

We now turn a description of the effect in the framework of the Boltzmann kinetic equation. Due to the rapid mixing of rotational degrees of freedom of the molecules, their distribution function depends only on the center of 
mass coordinate $\mathbf{r}$, time $t$, and the set of quantities $\Gamma$ that are conserved during free motion [10. Below we consider a spatially-uniform situation and assume that the chiral particles scatter only from non-chiral buffer gas, whose distribution function is in equilibrium. Then the Boltzmann kinetic equation has the form

$$
\frac{\partial \tilde{f}^{a}(\Gamma)}{\partial t}=I^{a}\{\tilde{f}(\Gamma)\}+I_{p h}^{a}(\Gamma) .
$$

Here $\tilde{f}^{a}(\Gamma)$ is the nonequilibrium part of the distribution function, and $a=g, e$ labels the ground or exited states of the chiral molecules. Denoting the scattering probability of a molecule from state $a, \Gamma$ to $a^{\prime}, \Gamma^{\prime}$ by $w^{a^{\prime} a}\left(\Gamma^{\prime} ; \Gamma\right)$ we write the scattering integrals in the excited and ground state due to collisions with the buffer gas as

$$
\begin{aligned}
I^{g}\{\tilde{f}(\Gamma)\}= & \int w^{g g}\left(\Gamma ; \Gamma^{\prime}\right) \tilde{f}^{g}\left(\Gamma^{\prime}\right) d \Gamma^{\prime} \\
& -\tilde{f}^{g}(\Gamma) \int w^{g g}\left(\Gamma^{\prime} ; \Gamma\right) d \Gamma^{\prime} \\
& +\int w^{g e}\left(\Gamma ; \Gamma^{\prime}\right) \tilde{f}^{e}\left(\Gamma^{\prime}\right) d \Gamma^{\prime}, \\
I^{e}\{\tilde{f}(\Gamma)\}= & -\tilde{f}^{e}(\Gamma) \int w^{g e}\left(\Gamma^{\prime} ; \Gamma\right) d \Gamma^{\prime} .
\end{aligned}
$$

To linear order in the light intensity $\mathcal{I}$ the transition rates between the ground and exited states due to light absorption are described by

$$
\begin{aligned}
I_{p h}^{g}(\Gamma) & =-f_{0}(\Gamma) \Upsilon(\Gamma) \mathcal{I}, \\
I_{p h}^{e}(\Gamma) & =f_{0}(\Gamma) \Upsilon(\Gamma) \mathcal{I},
\end{aligned}
$$

where $f_{0}(\Gamma)$ is the equilibrium distribution function of the molecules in the ground state (we assume that the excited state is not thermally populated), and $\Upsilon(\Gamma)$ is the probability of light absorption. The latter is determined by the time dependence of the electric field in the reference frame of the molecule, and in general depends both on the molecule velocity (due to the Doppler effect) and its angular velocity. The rotational frequency shift is of order of the angular velocity of rotation, $\sim v_{T} / d$, where $v_{T}$ is the thermal speed of the molecule and $d$ is its characteristic size. The Doppler frequency shift is much smaller, of order $v_{T} / \lambda$, where and $\lambda$ is the wavelength of light. We assume that the mismatch between the radiation frequency $\omega$ and the resonant frequency $\omega_{0}$ is of the order of the thermal rotational shift, i.e. $\left|\omega-\omega_{0}\right| \sim v_{T} / d$, and that the absorption line width $\gamma$ is smaller than the rotational broadening $\gamma \ll v_{T} / d$. In this regime the Doppler effect can be neglected.

Thus we take the absorption coefficient $\Upsilon(\Gamma)$ to be independent of the linear momentum of the chiral molecules. Since we work in the dipole approximation, in which $\Upsilon(\Gamma)$ is insensitive to the molecule chirality it is invariant under inversion. In other words $I_{p h}^{a}(\Gamma)=I_{p h}^{a}\left(\Gamma^{P}\right)$, where the set of coordinates $\Gamma^{P}$ is obtained from $\Gamma$ by inversion. If the molecules were nonchiral the scattering probabilities would be invariant under inversion, i.e.

$$
w\left(\Gamma^{\prime} ; \Gamma\right)=w\left(\Gamma^{\prime P} ; \Gamma^{P}\right)
$$

In this case the nonequilibrium part of the distribution function $\tilde{f}(\Gamma)$ would be a symmetric function of momentum, $\tilde{f}(\Gamma)=\tilde{f}\left(\Gamma^{P}\right)$. This would nullify the chiral current

$$
\mathbf{j}_{c}=\sum_{a} \int d \Gamma \mathbf{v} \tilde{f}^{a}(\Gamma),
$$

where $\mathbf{v}$ is the molecule velocity. For chiral molecules, in addition to inverting velocity, the inversion symmetry changes the molecule chirality. In this situation the equality Eq. (5) is not valid, and the nonequilibrium distribution $\tilde{f}(\Gamma)$ contains a part that is odd in momentum and the chiral current Eq. (6) generally does not vanish.

In order to complete the description of kinetics of the molecules in the presence of radiation we need to obtain the dependence of the absorbtion probability on the phase space coordinates, $\Upsilon(\Gamma)$. In the dipole approximation $\Upsilon(\Gamma)$ can be expressed in terms of the three frequency dependent absorbtion cross-section, $\sigma_{i}(\omega)$, for the different light polarizations, by transforming the electric field from the lab frame to the reference frame co-rotating with the molecule. This problem can be solved for the general case, where molecule rotation is described by an asymmetric top [11. However, in order to simplify the discussion and illustrate the essential phisics we consider the case where the molecule rotation is modeled by a symmetric top.

In this case the set of conserved quantities consists of the linear momentum $\mathbf{p}$, angular momentum $\mathbf{M}$, and the angle $\theta$ between the molecular axis and $\mathbf{M}$, i.e. $\Gamma=\{\mathbf{p}, \mathbf{M}, \theta\}$ (we introduce the Euler angles $\phi, \theta$, and $\psi$, as shown in Fig. 2 b)) 20. In the case of circularly polarized light the distribution function depends not on all three components of the angular momentum $\mathbf{M}$ but only on its magnitude $M$ and the angle $\beta$ it makes with the direction of light propagation ( $z$ axis in our notations, see Fig. 2 a)). Thus the distribution function $\tilde{f}$ depends only on $\mathbf{p}, M, \theta, \beta$. Furthermore, it is convenient to express $M$ and $\theta$ in terms of the components of the angular momentum parallel, $M_{3}=M \cos \theta$, and perpendicular, $M_{\perp}=M \sin \theta$, to the symmetry axis of the molecule. Indeed the rotational energy of a symmetric top is most naturally expressed in these variables; $\varepsilon(\Gamma)=\frac{M_{3}^{2}}{2 I_{3}}+\frac{M_{\perp}^{2}}{2 I_{\perp}}$. Therefore below we write the equilibrium distribution function and the kinetic equation in the variables $\Gamma=\left\{\mathbf{p}, M_{3}, M_{\perp}, \beta\right\}$, with the integration measure $d \Gamma \sim d^{3} p d \cos \beta M_{\perp} d M_{\perp} d M_{3}$.

We assume that the excited state is non-degenerate. In this case, for a symmetric top molecule and in the dipole approximation, only the electric field polarized along the 



FIG. 2: (a) Rotation by the angle $\beta$ from the $x y z$ frame to the $\xi \eta \zeta$ frame. (b) Illustration of the Euler angles $\theta, \phi, \psi$ rotating the $\xi \eta \zeta$ frame to the $x_{1} x_{2} x_{3}$ frame co-rotating with the molecule.

symmetry axis, $x_{3}$, of the molecule is absorbed. Furthermore, the absorption cross-section for the right and left stereo-isomers are identical and will be denoted by $\sigma(\omega)$.

In order to express $\Upsilon(\Gamma)$ in terms of $\sigma(\omega)$ we need to transform the electromagnetic field $\mathbf{E}$ from the stationary frame to the frame rotating with the molecule. To this end we introduce three reference frames as illustrated in Fig. 2. The $x y z$ frame is the lab frame such that the $z$-axis corresponds to the direction of light propagation. The light is assumed circularly polarized with the polarization vector $\mathbf{E}=E_{0}(\hat{x}+i \hat{y}) / \sqrt{2}$, where the hat above a symbol is used to denote a unit vector along the corresponding axis. The $\xi \eta \zeta$ frame is also stationary but the $\zeta$ axis points along the constant angular momentum $\mathbf{M}$ of a given molecule and forms an angle $\beta$ with the $z$-axis. The $\xi$ and the $x$ axes coincide. In terms of the basis vectors $\hat{\xi}, \hat{\eta}, \hat{\zeta}$ of this frame the electric field is written as

$$
\mathbf{E}=E_{+} \frac{\hat{\xi}+i \hat{\eta}}{\sqrt{2}}+E_{\zeta} \hat{\zeta}+E_{-} \frac{\hat{\xi}-i \hat{\eta}}{\sqrt{2}}
$$

with the components expressed in terms of $E_{0}$ as

$$
\left(E_{+}, E_{\zeta}, E_{-}\right)=E_{0}\left(\frac{1+\cos \beta}{2}, \frac{-i \sin \beta}{\sqrt{2}}, \frac{1-\cos \beta}{2}\right) .
$$

Next we transform the electric field from the fixed frame $\xi \eta \zeta$ to the frame $x_{1} x_{2} x_{3}$ which rotates with the molecule so that the $x_{3}$-axis always point along the symmetry axis of the molecule. The rotation from the $\xi \eta \zeta$ frame to the $x_{1} x_{2} x_{3}$ frame is described by the Euler angles $\phi, \theta, \psi$.

The components of the electric field in the rotating frame, $\mathbf{E}=\tilde{E}_{+} \frac{\hat{x}_{1}+i \hat{x}_{2}}{\sqrt{2}}+\tilde{E}_{0} \hat{x}_{3}+\tilde{E}_{-} \frac{\hat{x}_{1}-i \hat{x}_{2}}{\sqrt{2}}$, are given by

$$
\left(\begin{array}{c}
\tilde{E}_{+} e^{-i \psi} \\
\tilde{E}_{0} \\
\tilde{E}_{-} e^{i \psi}
\end{array}\right)=\left(\begin{array}{ccc}
\frac{1+\cos \theta}{2} & \frac{-i \sin \theta}{\sqrt{2}} & \frac{1-\cos \theta}{2} \\
\frac{-i \sin \theta}{\sqrt{2}} & \cos \theta & \frac{i \sin \theta}{\sqrt{2}} \\
\frac{1-\cos \theta}{2} & \frac{i \sin \theta}{\sqrt{2}} & \frac{1+\cos \theta}{2}
\end{array}\right)\left(\begin{array}{c}
E_{+} e^{i \phi} \\
E_{\zeta} \\
E_{-} e^{-i \phi}
\end{array}\right) \text {. }
$$

For a symmetric top molecule the angles $\phi$ and $\psi$ change linearly with time while the angles $\beta$ and $\theta$ do not change during free propagation. Thus it is clear from Eq. (8) that in the reference frame co-moving with the molecule each component of the electric field, $\tilde{E}_{ \pm}$, and $\tilde{E}_{0}$, consists of three monochromatic waves with frequencies of the form

$$
\omega_{p q} \equiv \omega-p \dot{\psi}-q \dot{\phi},
$$

where $p$ and $q$ can take the values $p, q=0, \pm 1$. Since only the field $\tilde{E}_{0}$ along the molecule axis $x_{3}$ is absorbed, we can write the absorption probability using Eq. (8) as

$$
\begin{aligned}
\Upsilon(\Gamma)= & \frac{1}{\hbar \omega}\left[\frac{\sin ^{2} \theta}{8} \sum_{q= \pm 1}(1+q \cos \beta)^{2} \sigma\left(\omega_{0 q}\right)\right. \\
& \left.+\frac{\cos ^{2} \theta \sin ^{2} \beta}{2} \sigma\left(\omega_{00}\right)\right] .
\end{aligned}
$$

The frequencies $\omega_{p q}$ can be expressed in terms of the variables $M_{3}$ and $M_{\perp}, M=\sqrt{M_{\perp}^{2}+M_{3}^{2}}$, using the kinematic relations

$$
\dot{\psi}=\frac{M_{3}}{I_{3}}-\frac{M_{3}}{I_{\perp}}, \quad \dot{\phi}=\frac{M}{I_{\perp}}, \quad \cos \theta=\frac{M_{3}}{M} .
$$

Equations (10, (9) and (11) define the absorption probability as a function of the phase space coordinates $\Gamma=\left\{\mathbf{p}, M_{3}, M_{\perp}, \beta\right\}$. This probability is peaked when the resonance condition $p \dot{\psi}+q \dot{\phi}=\omega-\omega_{0}$ is satisfied. In terms of the variables $M_{3}, M_{\perp}$ the resonance condition is

$$
p M_{3}\left(\frac{I_{\perp}}{I_{3}}-1\right)+q \sqrt{M_{3}^{2}+M_{\perp}^{2}}=I_{\perp}\left(\omega-\omega_{0}\right) .
$$

We note that the light absorbtion probability Eq. 10p contains a term that is linear in $\cos \beta$. The coresponding optical transitions produce a nonequilibrium distribution function that is odd in the component of the angular momentum along the light propagation direction, $M_{z}$. Subsequent collisions of the chiral molecules with the buffer gas result in a net flux of the molecules along the $z$-axis.

In order to obtain an estimate for the magnitude of the effect we consider the simplest case when the relaxation time $\tau_{e g}$ from excited to ground state is the shortest time in the problem. Here $1 / \tau^{g e}(\Gamma)=\int d \Gamma^{\prime} w^{g e}\left(\Gamma, \Gamma^{\prime}\right)$. Then in a stationary state we get from Eqs. (2), (3b) and 4b

$$
\tilde{f}^{e}(\Gamma)=\tau^{g e}(\Gamma) \mathcal{I} \Upsilon(\Gamma) f_{0}^{g}(\Gamma),
$$

while the Boltzmann equation for the ground state distribution function becomes

$$
\begin{aligned}
& \tilde{f}^{g}(\Gamma) \int d \Gamma^{\prime} w^{g g}\left(\Gamma^{\prime} ; \Gamma\right)-\int d \Gamma^{\prime} w^{g g}\left(\Gamma ; \Gamma^{\prime}\right) \tilde{f}^{g}\left(\Gamma^{\prime}\right)= \\
& \mathcal{I}\left[-\Upsilon(\Gamma) f_{0}^{g}(\Gamma)+\int d \Gamma^{\prime} w^{g e}\left(\Gamma ; \Gamma^{\prime}\right) \tau^{g e}\left(\Gamma^{\prime}\right) \Upsilon\left(\Gamma^{\prime}\right) f_{0}^{g}\left(\Gamma^{\prime}\right)\right] .
\end{aligned}
$$

In our approximation the molecules in the excited state do not contribute to the total flux of chirality, i.e.

$$
\mathbf{j}_{c}=\int \mathbf{v} \tilde{f}^{g}(\Gamma) d \Gamma .
$$


Thus we are interested only in the anisotropic in $\mathbf{v}$ part of $\tilde{f}^{g}(\Gamma)$, which can be estimated as

$$
\begin{aligned}
\mathbf{j}_{c} \sim & \tau_{p}^{g g} \mathcal{I} \int d \Gamma d \Gamma^{\prime} \mathbf{v} \Upsilon\left(\Gamma^{\prime}\right) f_{0}^{g}\left(\Gamma^{\prime}\right) \times \\
& {\left[w^{g e}\left(\Gamma, \Gamma^{\prime}\right) \tau^{g e}\left(\Gamma^{\prime}\right)-w^{g g}\left(\Gamma, \Gamma^{\prime}\right) \tau^{g g}\left(\Gamma^{\prime}\right)\right] . }
\end{aligned}
$$

Here we introduced the relaxation time for scattering of chiral molecules in the ground state, $\tau^{g g}$, and the momentum relaxation time, $\tau_{p}^{g g}$.

A rough estimate for the chiral current Eq. 13 can be written as

$$
\begin{array}{r}
j_{c} \sim v_{T} n_{c}\left(B \tau_{p}\right)\left(\delta W^{g e} \tau^{g e}-\delta W^{g g} \tau^{g g}\right) \approx \\
v_{T} \frac{I \tau_{p}}{\xi \hbar \omega}\left(\delta W^{g e} \tau^{g e}-\delta W^{g g} \tau^{g g}\right)
\end{array}
$$

Here $n_{c}$ is the concentration of the chiral molecules, $v_{T}$ is their thermal velocity, $\xi$ is the absorption length of the light, and $B \sim n_{c}^{-1} \mathcal{I} \int d \Gamma \Upsilon(\Gamma) f_{0}(\Gamma)$ is the rate of the optical transitions in an individual molecule. Here we introduced the "chiral part" of the scattering rate as $\delta W^{g a} \sim\left(B n_{c} v_{T}\right)^{-1} \int d \Gamma d \Gamma^{\prime} \hat{z} \cdot \mathbf{v}\left[w^{g a}\left(\Gamma ; \Gamma^{\prime}\right)-\right.$ $\left.w^{g a}\left(\Gamma^{P} ; \Gamma^{\prime P}\right)\right] f_{0}^{g}\left(\Gamma^{\prime}\right) \Upsilon\left(\Gamma^{\prime}\right) \mathcal{I}$, with $a=e / g$. Thus $\delta W^{g a} \tau^{g a}$ is a dimensionless measure of the degree of chirality of the molecules (we are not aware of any studies of this quantity). Equation (14) holds at small enough intensities of radiation, when $f \ll f_{0}$. At large intensities the magnitude of the effect saturates, and the maximum flux of chiral molecules is of order

$$
j^{(\max )} \sim v_{T} n_{c}\left(\frac{\gamma}{T}\right)\left(\delta W^{g e}-\delta W^{g g}\right) \tau_{p}^{g g} .
$$

We note that relative signs of $\delta W^{g e}$ and $\delta W^{g g}$, as well as the sign of the chiral current, are arbitrary and depend on the structure of the molecules.

The effects considered above are direct analogues of photo-galvanic effects in non-centrosymmetric crystals. There are two types of photo-galvanic effects. The first one is associated with a transfer of momentum from light to electrons, and its magnitude is proportional to the momentum of light. The analogue of this effect, which is associated with transfer of angular momentum of electromagnetic field to molecules in classical gases and liquids has been considered in Refs. 12, 13, 14, 15. The second type of the photo-galvanic effects exist only in noncentrosymmetric crystals and is not proportional to the momentum of light [16, 17, 18, 19]. Since the momentum of light is small the magnitude of this effect is generally bigger than that of the first one. The photo-induced drift of chiral molecules diluted in a buffer gas considered here, is an analogue of the second type of photo-galvanic effect.

There exists also an analogue of the linear photogalvanic effect [16, 17, 18, 19]. Namely, in the presence of a magnetic field a flux of chiral particles exists even if the electromagnetic field is linearly polarized or unpolarized,

$$
j_{c, k}=\tilde{\alpha}_{c} H_{k} P_{j j}+\tilde{\beta}_{c}\left(P_{k j}+P_{j k}\right) H_{j} .
$$

The photo-induced chiral drift also exists when chiral molecules are dissolved in a liquid. Photo-induced propulsion of molecules in this situation can be described in the framework of hydrodynamics. These two aspects of the phenomenon will be considered in a separate publication.

We acknowledge useful discussions with A. Grosberg, E. Ivchenko, B. Shklovskii, D. Son and G. Tartakovski. The work was supported by NSF grant DMR-0704151 and DOE grant DE-FG02-07ER46452.

[1] S. Saha, J. F. Stoddart, Chem. Soc. Rev. 36, 77, (2007).

[2] M. K. J. Wiel at all, Org. Biomol. Chem. 3, 4071, (2005).

[3] Nagotoshi Koumura et al, Nature, 401, 152, (1999).

[4] T. R. Kelly, H. De Silva, R. A. Silva, Nature, 401, 150, (1999).

[5] M. M. Polland, M. Klok, D. Pijer, B. L. Feringa, Adv. Funct. Mater. 17, 718, (2007).

[6] F. Kh. Gelmikhanov, A. M. Shalagin, JETP Lett. 29 771, (1979).

[7] A. M. Dykhne, A. N. Starostin, Sov. Phys. JETP. 52, 612, (1980).

[8] H. G. C. Werij, J. E. M. Haverkort, J. P. Woerdman, Phys. Rev. A 33 3270, (1986), M. C. de Lignie, J. P. Woerdeman, J. Phys. B: At. Mol. Opt. Phys. 23, 417, (1990).

[9] I. Kuscer, L. J. F. Hermans, P. L. Chaplovsky, J. J. M. Beenakker, G. J. van der Meer, J. Phys. B: At. Mol. Opt. Phys. 26, 2837, (1993).

[10] E. M. Lifshitz, L. P. Pitaevskii, Physical Kinetics (Butterworth-Heinemann, 1999).

[11] L. D. Landau and E. M. Lifshitz, Mechanics, (Butterworth-Heinemann, 2001).

[12] N. B. Baranova, B. Ya. Zel'dovich, Chem. Phys. Lett. 57, 435 (1978).

[13] J. E. Curtis, D. E. Grier, Opt. Lett. 28, 872, (2003).

[14] H. He, N. R. Heckenberg, H. Rubinsztein-Dnlop, J. Mod. Opt. 42, 217, (1995).

[15] Y. Roichman, B. Sun, Y. Roichman, J. Amato-Grill and D. G. Grier, Phys. Rev. Lett. 100, 013602 (2008).

[16] V. I. Belinicher, Phys. Lett. A 66, 213 (1978).

[17] B. I. Sturman, V. M. Fridkin, The Photovoltaic and Photorefractive effects in Noncentrosymmetric Materials, Gordon and Breach Science Publishers, 1992.

[18] E. L. Ivchenko and G. E. Pikus, Zh. Exsp. Teor. Phys. Pisma 27, 640 (1978) [JETP Lett. 28, 74 (1978)].

[19] E. L. Ivchenko and G. E. Pikus, Superlattices and Other Heterostructures. Symmetry and Optical Phenomena, Springer Series in Solid State Sciences, vol. 110, SpringerVerlag, 1997.

[20] As was mentioned, because of the fast change of the angles $\psi$ and $\phi$ the distribution function of chiral molecules depends only on the conserved angle $\theta$ between the molecule axis and the angular momentum. 